\title{
Multidimensional intervention in individuals with mild cognitive impairment: a pilot nonrandomized study
}

\author{
Shaofang Xu ${ }^{1}$, Guilin Meng ${ }^{1}$, Pengfei Chen ${ }^{1}$, Min Fang ${ }^{2}$, Aiping Jin ${ }^{1}$ \\ ${ }^{1}$ Neurological Rehabilitation Center, Department of Neurology, Shanghai Tenth People's Hospital, Tongji University School of Medicine, Shanghai, \\ China; ${ }^{2}$ Department of Neurology, Shanghai Tenth People's Hospital, Tongii University School of Medicine, Shanghai, China \\ Contributions: (I) Conception and design: A Jin, M Fang; (II) Administrative support: A Jin; (III) Provision of study materials or patients: S Xu, M \\ Fang, G Meng; (IV) Collection and assembly of data: S Xu, P Chen; (V) Data analysis and interpretation: S Xu, G Meng; (VI) Manuscript writing: \\ All authors; (VII) Final approval of manuscript: All authors. \\ Correspondence to: Aiping Jin. Neurological Rehabilitation Center, Department of Neurology, Shanghai Tenth People's Hospital, Tongji University \\ School of Medicine, 301 Yanchang Middle Road, Jing'an District, Shanghai 200072, China. Email: 13402140058@163.com; Min Fang. Department \\ of Neurology, Shanghai Tenth People's Hospital, Tongji University School of medicine, 301 Yanchang Middle Road, Jing'an District, Shanghai \\ 200072, China. Email: fangmin_dr@126.com.
}

Backgrounds There is an increasing need for non-pharmacological treatments that can enhance the cognitive function of individuals with mild cognitive impairment. We firstly performed multidimensional intervention based on the concept of the International Classification of Functioning, Disability and Health, and aimed to explore its short-term effect on the improvement of cognitive function in individuals with mild cognitive impairment.

Methods: Twenty-four individuals with mild cognitive impairment in this pilot study were recruited from the memory clinic and neurology ward in Shanghai Tenth People's Hospital from August 2018 to August 2019. According to participants' personal wishes, 13 and 11 participants were enrolled into an intervention group and a control group, respectively. Based on the International Classification of Functioning, Disability and Health, we performed baseline assessments to all participants. According to the assessment results and the wishes and hobbies of the patients, then doctors, therapists, nurses, patients and their families together chose the appropriate multidimensional interventions to the intervention group in seven 1-hour sessions and health education to the both groups. After one week, all participants underwent reevaluation of cognitive function.

Results: There were significant differences between the two groups on the improvement of cognitive

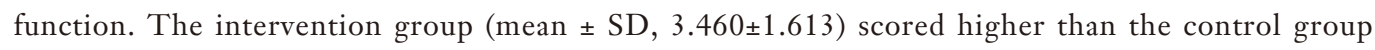
$(1.360 \pm 0.924)$ on the change score of the total score in the Montreal Cognitive Assessment $(t=3.808$, $\mathrm{P}<0.001$, 95\% CI: 0.955-3.240), though not on the change score of any cognitive domain score. Regression results showed that the change score of the total score was negatively correlated with the baseline score of Abstraction score $(\mathrm{aR} 2=0.583, \beta=-0.506, \mathrm{P}=0.031)$ and the modified Barthel index score $(\beta=-0.464$, $\mathrm{P}=0.045)$ in the intervention group.

Conclusions: The pilot study demonstrated that the short-term multidimensional intervention may produce cognitive benefits in individuals with mild cognitive impairment.

Keywords: International Classification of Functioning, Disability and Health (ICF); mild cognitive impairment; multidimensional intervention; short-term effect

Submitted Feb 09, 2020. Accepted for publication Sep 18, 2020.

doi: 10.21037/apm-20-346

View this article at: http://dx.doi.org/10.21037/apm-20-346 


\section{Introduction}

Mild cognitive impairment (MCI) is a disorder in which individuals demonstrate cognitive impairment with minimal disruption to instrumental activities $(1,2)$. Nonpharmacological treatments in MCI have been of great concern.

Recently, cognitive training and cognitive rehabilitation have become important non-pharmacological therapeutic approaches for MCI. For example, cognitive training describes a group of games that exercise mental processing abilities including attention, memory, calculation, etc. (3). Routine cognitive rehabilitation is to use strategies or interventions to manage or reduce difficulties most relevant to the individuals with cognitive impairment (4). However, these effects of cognitive trainings or routine cognitive rehabilitations in individuals with MCI are often not observed in the short-term which affects patient enthusiasm and compliance for training or rehabilitation, and, furthermore, low compliance in turn may weaken these effects. In addition, cognitive trainings or routine cognitive rehabilitations just focus on cognitive function and/or some of the functions related to cognitive function. Besides mental exercise, MCI patients need further exercises based on the concept of the International Classification of Functioning, Disability and Health (ICF) (5), such as physical exercises, relaxation training, health instruction, etc.

The ICF was propounded by the World Health Organization, and it provides a comprehensive framework for evaluation (5); all rehabilitation interventions are based on the evaluation, and the accurate evaluation helps us to choose accurate and effective interventions, so ICF also provides a comprehensive framework for intervention. Maki et al. suggested interventions that enable the wellbeing of dementia patients should be performed using the framework of ICF (6). ICF is grounded in the concept of assessing health condition through the perspective of the whole person. It comprises of three components (Body functions and structures, Activity, and Participation) and two contextual factors (Environmental and Personal), all of which are related (5) (Figure 1). The symptoms of MCI include impairments of different functions, such as cognitive impairment, difficulties undertaking complex activities in daily living (7), and changes in mood and behavior (8). Since these symptoms and other contextual factors are mutually related, we proposed that the intervention for MCI patients should be multidimensional.

Therefore, in the current pilot study, we firstly performed multidimensional intervention and aimed to explore whether the intervention could significantly improve cognitive function in MCI patients. Due to the comprehensiveness and multidimension of the intervention, we explore whether its effect will be observed as early as one week after commencement. We present the following article in accordance with the TREND Statement reporting checklist (available at http://dx.doi.org/10.21037/apm-20-346).

\section{Methods}

\section{Participants}

Twenty-four patients with MCI were recruited from the memory clinic and neurology ward in Shanghai Tenth People's Hospital from August 2018 to August 2019. According to participants' personal wishes, 13 and 11 participants were enrolled into an intervention group and a control group, respectively. There were no significant differences between the two groups in clinical characteristics (Table 1) and baseline total scores and cognitive domains scores on the Montreal Cognitive Assessment (MoCA) and the Mini-mental State Examination (MMSE) (Table 2). In this study, the probable etiological subtypes of MCI included mild neurocognitive disorder due to Alzheimer's disease and mild neurocognitive disorder owing to multiple etiologies (e.g., Alzheimer's disease and vascular neurocognitive disorder). The study protocol was approved by the Ethics Committee of Shanghai Tenth People's Hospital (No. SHSY-IEC-4.1/19-124/01) and conforms to the provisions of in accordance with the Helsinki Declaration as revised in 2013. All participants gave written informed consent.

\section{Inclusion criteria}

In the study, participants met all the following criteria: (I) accorded with the MCI diagnosis criteria of the Diagnostic and Statistical Manual of Mental Disorders, Fifth Edition (9); (II) had no history of severe psychiatric disease, or drug, and/or alcohol abuse; (III) were not currently taking neuroactive drugs (prescribed low-dose sleep medication was an exception); and (IV) had a Functional Activities Questionnaire (FAQ) score between zero and five.

\section{Exclusion criteria}

Participants who met any of the following criteria were 


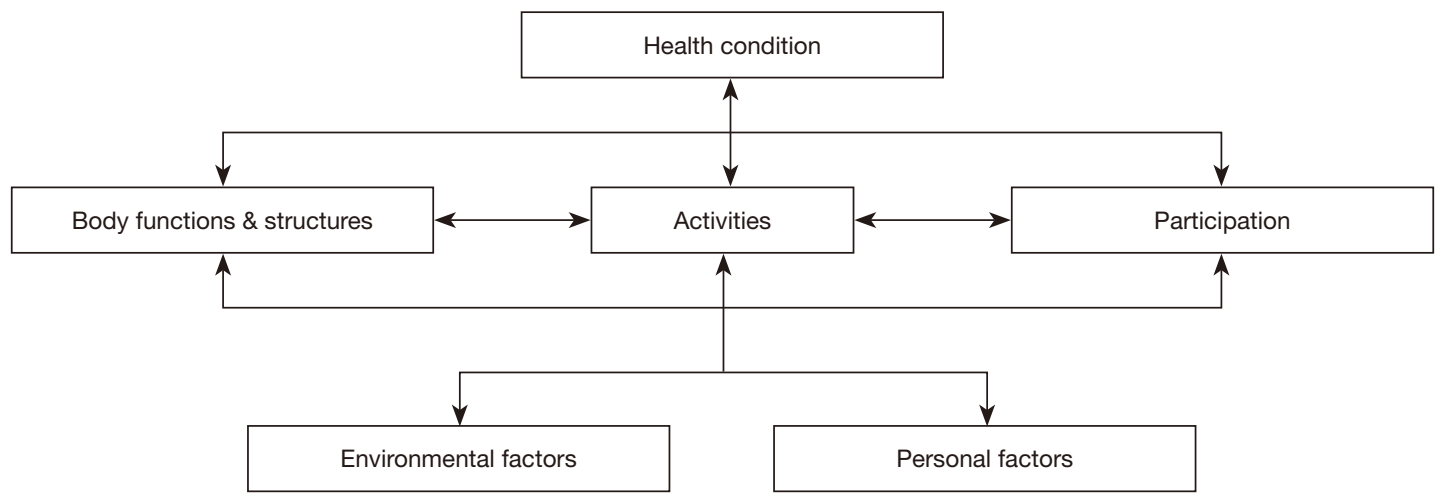

Figure 1 Concept of International Classification of Functioning, Disability and Health. The International Classification of Functioning, Disability and Health aims to assessing health condition through the perspective of the whole person.

excluded from the study: (I) active psychiatric disorders; (II) acute cerebrovascular disorders; (III) substance abuse disorders; (IV) other forms of dementia; or (V) cognitive impairment with significant impact on functional independence based on the clinical interview; (VI) FAQ score > five; and (VII) Hamilton Depression Scale (HAMD)/17-item score $>17$, and Hamilton Anxiety Scale (HAMA) score $\geq 14$.

\section{Baseline assessment}

Based on the ICF, we performed baseline assessments including Body functions \& structures and Activities, and we got information about Participation, Environmental factors and Personal factors. Baseline Body functions \& structures and Activities clinical characteristics included age, sex, education, probable etiology, marital status, cognitive disorder history, course of disease, neurological function (vision, hearing, speech, obstructive sleep apnea syndrome, walking, numbness, tingling), modified Barthel index (MBI), FAQ, HAMA, HAMD, MoCA and MMSE. Neuropsychological measures were obtained by neuropsychological assessors who were blind to grouping.

\section{Cognitive measures}

The Beijing version of the MoCA translated from the original English was applied in the current study (10), and it involves seven cognitive domains (Visuospatial/executive function, Naming, Attention, Abstraction, Language, Delayed memory, and Orientation). The Chinese version of the MMSE was used to detect the dementia in the Chinese population (11), and the MMSE covers five domains (12):
Orientation of place and time, Registration, Attention and calculation, Recall, Language and visual construction.

\section{Cognitive rebabilitation}

Based on the International Classification of Functioning, Disability and Health, we performed baseline assessments to all participants. According to the assessment results and the wishes and hobbies of the patients, then doctors, therapists, nurses, patients and their families together chose the appropriate multidimensional interventions to the intervention group in seven 1-hour sessions and health education to the both groups. The multidimensional intervention consisted of individualized physical therapy, occupational therapy, speech therapy, rehabilitation psychology, rehabilitation engineering and educational information. Educational information pertained to MCI in terms of epidemiology, etiology, clinical manifestations and prognosis, and it also included recommendations for appropriate participation in social activities. Rehabilitation engineering suggestions involved in environmental and facilities reconstruction. Rehabilitation psychology included abdominal breathing relaxation training, listening and empathy, as well as other techniques to alleviate patients' and caregivers' psychological pressure. Speech therapy included memory, calculation, attention, visuospatial, orientation, executive skills, and so on. Occupational therapy included some strategies or trainings to manage or reduce difficulties most relevant to the participants. Physical therapy recommended some aerobic exercises. Professional therapists instructed patients to conduct their respective rehabilitation programs at neurological rehabilitation center and guaranteed the quality of the patient's rehabilitation 
Table 1 Participant clinical characteristics

\begin{tabular}{|c|c|c|c|}
\hline Characteristic & Intervention group & Control group & $P$ value ${ }^{a}$ \\
\hline Education (years), mean \pm SD & $10.230 \pm 3.086$ & $11.360 \pm 2.942$ & 0.368 \\
\hline Course of disease (years), mean \pm SD & $1.389 \pm 1.632$ & $3.278 \pm 3.874$ & 0.123 \\
\hline $\operatorname{Sex}(M / F), n$ & $8 / 5$ & $10 / 1$ & 0.228 \\
\hline $\begin{array}{l}\text { Probable etiological subtypes (mild neurocognitive disorder due to } \\
\text { Alzheimer's disease /multiple etiologies), } \mathrm{n}\end{array}$ & $3 / 10$ & $2 / 9$ & 0.865 \\
\hline Cognitive disorder history (yes/no), $\mathrm{n}$ & $0 / 13$ & $0 / 11$ & 1 \\
\hline Normal vision (yes/no), $\mathrm{n}$ & $13 / 0$ & $11 / 0$ & 1 \\
\hline Normal walking (yes/no), n & $12 / 1$ & $9 / 2$ & 0.691 \\
\hline Numbness (yes/no), n & $0 / 13$ & $0 / 11$ & 1 \\
\hline Tingling (yes/no), n & $0 / 13$ & $0 / 11$ & 1 \\
\hline $\mathrm{MBI}$, mean $\pm \mathrm{SD}$ & $96.460 \pm 12.758$ & $96.910 \pm 3.562$ & 0.912 \\
\hline $\mathrm{FAQ}$, mean $\pm \mathrm{SD}$ & $2.150 \pm 1.144$ & $2.270 \pm 1.009$ & 0.792 \\
\hline HAMA, mean \pm SD & $6.380 \pm 3.664$ & $6.180 \pm 4.332$ & 0.902 \\
\hline $\mathrm{HAMD}$, mean $\pm \mathrm{SD}$ & $7.460 \pm 4.484$ & $6.180 \pm 5.115$ & 0.520 \\
\hline
\end{tabular}

a, $\mathrm{P}$ value for analysis of variance group effect. None of the measures had a significant group effect. MBI, modified Barthel index; FAQ, Functional Activities Questionnaire; HAMA, Hamilton Anxiety Scale; HAMD, Hamilton Depression Scale.

interventions. After each session, the participants were given homework to strengthen the interventions.

\section{Health education}

Health education was given to both groups by doctors, therapists, nurses. It included educational information pertained to MCI in terms of epidemiology, etiology, clinical manifestations and prognosis, and it also included recommendations for appropriate participation in social activities.

\section{Cognitive change}

Participants underwent reevaluation on the MoCA and MMSE scales after one week. Evaluators were blinded to baseline results and were not involved in implementing any aspect of the intervention. Change score was defined to reevaluation score minus baseline score. Change rate of the score was defined to change score divided by baseline score.

\section{Sample size calculation and statistical analysis}

Sample size calculation for comparison between two groups was performed. We selected type 1 error rate at $5 \%$ and the power of study at $80 \%$. The endpoint is the change score of the total score on the MoCA, the sample sizes is 7 subjects per group.

The IBM SPSS statistics was used to perform Statistical analyses. We used the Kolmogorov-Smirnov test to determine the normality of distribution for all scale variables. A $t$-test was used to test for the differences about the normally distributed variables between the two groups and to estimate the $95 \%$ confidence interval (CI) for differences. Mann-Whitney test was performed to test for the differences about the non-normally distributed variables 
Table 2 Baseline scores and change scores on the MoCA and MMSE scales in two groups

\begin{tabular}{|c|c|c|c|}
\hline & Intervention group & Control group & $P$ value \\
\hline Total score & $20(16.500-25)$ & $22(20-23)$ & 0.683 \\
\hline Visuospatial/executive function & $3(2.500-4.500)$ & $4(2-5)$ & 0.767 \\
\hline Naming & $3(2-3)$ & $3(2-3)$ & 0.351 \\
\hline Abstraction & $1(0-2)$ & $1(1-2)$ & 0.779 \\
\hline Language & $1(0-2)$ & $1(1-2)$ & 0.303 \\
\hline Delayed memory & $2(0-3)$ & $1(0-2)$ & 0.512 \\
\hline Orientation & $6(5-6)$ & $6(6-6)$ & 0.059 \\
\hline Orientation & $9(8-10)$ & $10(10-10)$ & 0.099 \\
\hline Registration & $3(2.500-3)$ & $3(3-3)$ & 0.096 \\
\hline Attention and calculation & $5(3-5)$ & $5(3-5)$ & 0.655 \\
\hline Recall & $2(0-2)$ & $1(0-2)$ & 0.394 \\
\hline Language and visual construction & $8(6-9)$ & $8(6-9)$ & 0.382 \\
\hline \multicolumn{4}{|l|}{ Change score on the MoCA } \\
\hline Total score & $3.460 \pm 1.613$ & $1.360 \pm 0.924$ & $<0.001$ \\
\hline Visuospatial/executive function & $0(0-1)$ & $0(0-1)$ & 0.553 \\
\hline Orientation & $0(0-0.500)$ & 00 & 0.326 \\
\hline \multicolumn{4}{|l|}{ Change score on the MMSE } \\
\hline Total scores & $2.460 \pm 1.664$ & $0.730 \pm 1.679$ & 0.019 \\
\hline Orientation & $0(0-1)$ & $0(0-0)$ & 0.443 \\
\hline Registration & $0(0-0.500)$ & $0(0-0)$ & 0.191 \\
\hline Attention and calculation & $0(-0.500-1.500)$ & $0(0-1)$ & 0.601 \\
\hline Recall & $1(0-1)$ & $1(-1-1)$ & 0.730 \\
\hline Language and visual construction & $1(0-1.500)$ & $0(0-1)$ & 0.272 \\
\hline
\end{tabular}

Values are reported as median (25th-75th percentiles) for non-normal distribution data and as mean \pm SD for normally distributed data. Change score = reevaluation score minus baseline score. The MoCA scale involves seven cognitive domains: Visuospatial/executive function, Naming, Attention, Abstraction, Language, Delayed memory, and Orientation. The MMSE scale covers five domains: Orientation of place and time, Registration, Attention and calculation, Recall, Language and visual construction. 
between the two groups. The multiple linear regression with stepwise elimination was applied to investigate the impact of participant clinical characteristics and cognitive domains of the MoCA and MMSE scales on the change scores of the total scores and the change rates of the total scores about the MoCA and MMSE scales. A P value less than 0.05 was considered significant.

\section{Results}

\section{Cognitive change}

There were significantly different between the two groups on the change score of the MoCA total score $(t=-3.808$, $\mathrm{P}<0.001,95 \%$ CI: -3.240 to -0.955 ; the control group: $1.360 \pm 0.924$, the intervention group: $3.460 \pm 1.613$ ) (Table 2) , and the change score of the MMSE total score $(t=-2.534$, $\mathrm{P}=0.019$, 95\% CI: -3.154 to -0.315 ; the control group: $0.730 \pm 1.679$, the intervention group: $2.460 \pm 1.664$ ), although significant difference was not found on any cognitive domain of the MoCA and MMSE.

\section{Predicting response to multidimensional intervention}

We predicted the change scores of the total scores and the change rates of the total scores on the MoCA and MMSE scales based on clinical characteristics and baseline scores as well as the change scores of cognitive domains on the MoCA and MMSE scales. Regression results showed that the baseline score of Abstraction on the MoCA (change score of the total score: $\beta=-0.506, \mathrm{P}=0.031$; change rate of the total score: $\beta=-0.555, P=0.003)$ and the MBI score (change score of the total score: $\beta=-0.464, \mathrm{P}=0.045$; change rate of the total score: $\beta=-0.541, \mathrm{P}=0.003$ ) predicted the change score of the total score and the change rate of the total score on the MoCA in the intervention group. The change score of Registration $(\beta=0.858, \mathrm{P}<0.001)$, and the baseline scores of Attention and calculation $(\beta=-0.703$, $\mathrm{P}=0.001)$ on the MMSE predicted the change score of the total score on the MMSE in the intervention group, while the change scores of Recall ( $\beta=0.657, \mathrm{P}<0.001)$, Language and visual construction $(\beta=0.539, \mathrm{P}<0.001)$, Attention and calculation $(\beta=0.417, \mathrm{P}<0.001)$ and Registration $(\beta$ $=0.180, \mathrm{P}<0.001)$, and the baseline score of Orientation $(\beta$ $=-0.241, \mathrm{P}<0.001)$ on the MMSE did in the control group. The change score of Registration ( $\beta=0.586, \mathrm{P}=0.002$ ), the baseline scores of Attention and calculation $(\beta=-0.701$, $\mathrm{P}<0.001)$, as well as Language and visual construction ( $\beta$
$=-0.330, \mathrm{P}=0.042)$ on the MMSE, and the MBI score $(\beta$ $=-0.295, \mathrm{P}=0.024)$ predicted the change rate of the total score on the MMSE in the intervention group. The change scores of Recall $(\beta=0.820, P<0.001)$, Language and visual construction $(\beta=0.526, \mathrm{P}<0.001)$, Attention and calculation ( $\beta=0.486, P=0.002)$ on the MMSE and the HAMA score ( $\beta=-0.234, \mathrm{P}=0.033$ ) predicted the change rate of the total score on the MMSE in the control group (Table 3).

\section{Discussion}

We measured the cognitive effects after one-week of treatment with either multidimensional intervention or health education, in subjects with MCI. The study demonstrated that the short-term multidimensional intervention in patients with MCI may produce cognitive benefits.

In the present study, the group treated with multidimensional intervention improved on global cognitive abilities though not on specific cognitive domains. These results are consistent with those reported in other studies (13-16), indicating that cognitive rehabilitation may improve cognitive function in MCI. However, the effects on specific cognitive domains are inconsistent with different studies. For example, some studies have found significant improvements on executive function and/or memory $(13,16)$. Some researchers suggest that the efficacy of rehabilitation depends on the specificity of the training (17). Based on the ICF, our intervention considered the whole person, which may have led us to find significant improvements on global cognitive ability in the short-term, but its effect on specific cognitive domain may need more time.

We found the change scores of the total score and the change rates of the total score on the MoCA and MMSE scales were associated with HAMA score, MBI score and baseline scores as well as change scores of some cognitive domains on the MoCA and MMSE scales. Although the independent functioning of MCI is not precluded by cognitive deficits, some study supports that there are changes in MCI patients' psychological and daily functioning $(1,18,19)$. Neuropsychiatric symptoms are associated with MCI, with anxiety being one of the most common issues $(8,20)$. Moreover, the changes of daily functioning may be used to explore the probability of MCI patients developing into dementia $(19,21)$. A trial found that the cognitive function was ameliorated after a 3 -month rehabilitation training comprising physical and mental activity (22). Multi-domain defected MCI patients 
Table 3 Stepwise linear regression results about predicting the change scores and the change rates of the total scores on the MoCA and MMSE scales by participant clinical characteristics, the baseline scores and the change scores of cognitive domains scores

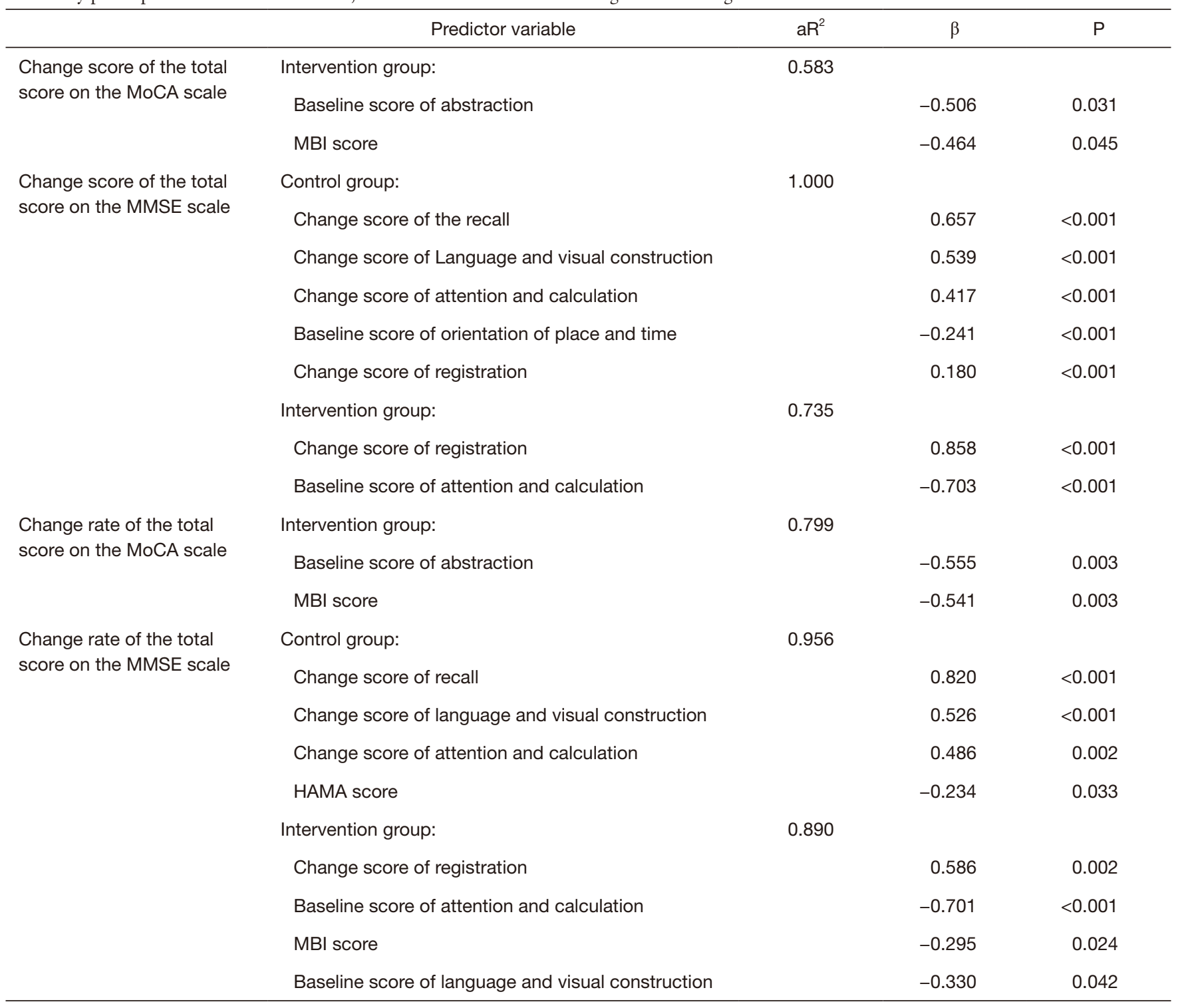

Only significant results are depicted. Change score $=$ reevaluation score minus baseline score. Change rate of the score $=($ reevaluation score - baseline score)/baseline score. The MoCA scale involves seven cognitive domains: Visuospatial/executive function, Naming, Attention, Abstraction, Language, Delayed memory, and Orientation. The MMSE scale covers five domains: Orientation of place and time, Registration, Attention and calculation, Recall, Language and visual construction. MBI, modified Barthel index. HAMA, Hamilton Anxiety Scale.

may experience more functional defects than those with single domain (19). Furthermore, cognitive, psychological and daily functioning are mutually related components in individuals with MCI, and therefore, it is beneficial to perform multidimensional assessment and multidimensional intervention.

There are some limitations in this study. First, the small sample size affected the statistical power and obstructed a more rigorous statistical analysis on the cognitive domains. Second, the multidimensional intervention was based on ICF, but the components of the multidimensional intervention were different among different people. Third, the study did not explore the relative contribution of rehabilitation components to the outcome. Finally, 
the study didn't explore the possible long-term effects of the multidimensional intervention and its effect on the participants' functional ability.

In future studies we aim to determine whether multidimensional intervention will lead to long-term improvement and/or preservation of functional ability in patients with MCI, in order to maintain quality-of-life in patients, delay the onset of dementia, and reduce the financial burden to patients, families and the healthcare system.

\section{Acknowledgments}

Funding: This study was supported by the Science and Technology Commission of Shanghai Municipality (17411950101; 18411961700; 19441908500) and Shanghai Municipal Key Clinical Specialty (shslczdzk06102).

\section{Footnote}

Reporting Checklist: The authors have completed the TREND Statement reporting checklist. Available at http:// dx.doi.org/10.21037/apm-20-346

Data Sharing Statement: Available at http://dx.doi. org/10.21037/apm-20-346

Conflicts of Interest: All authors have completed the ICMJE uniform disclosure form (available at http://dx.doi. org/10.21037/apm-20-346). The authors have no conflicts of interest to declare.

Ethical Statement: The authors are accountable for all aspects of the work in ensuring that questions related to the accuracy or integrity of any part of the work are appropriately investigated and resolved. The study protocol was approved by the Ethics Committee of Shanghai Tenth People's Hospital (No. SHSY-IEC-4.1/19-124/01) and conforms to the provisions of in accordance with the Helsinki Declaration as revised in 2013. All participants gave written informed consent.

Open Access Statement: This is an Open Access article distributed in accordance with the Creative Commons Attribution-NonCommercial-NoDerivs 4.0 International License (CC BY-NC-ND 4.0), which permits the noncommercial replication and distribution of the article with the strict proviso that no changes or edits are made and the original work is properly cited (including links to both the formal publication through the relevant DOI and the license). See: https://creativecommons.org/licenses/by-nc-nd/4.0/.

\section{References}

1. Petersen RC, Lopez O, Armstrong MJ et al. Practice guideline update summary: Mild cognitive impairment: Report of the Guideline Development, Dissemination, and Implementation Subcommittee of the American Academy of Neurology. Neurology 2018;90:126-35.

2. Langa KM, Levine DA. The diagnosis and management of mild cognitive impairment: a clinical review. JAMA 2014;312:2551-61.

3. Simons DJ, Boot WR, Charness $\mathrm{N}$ et al. Do "BrainTraining" Programs Work? Psychol Sci Public Interest 2016;17:103-86.

4. Woods B, Clare L. Clinical psychology of aging. Chichester: Wiley; 2008.

5. World Health Organization. The International Classification of Functioning, Disability and Health (ICF). WHO, Geneva, 2001.

6. Maki Y, Sakurai T, Okochi J et al. Rehabilitation to live better with dementia. Geriatr Gerontol Int 2018;18:1529-36.

7. Perneczky R, Pohl C, Sorg C et al. Complex activities of daily living in mild cognitive impairment: conceptual and diagnostic issues. Age Ageing 2006;35:240-5.

8. Apostolova LG, Cummings JL. Neuropsychiatric manifestations in mild cognitive impairment: a systematic review of the literature. Dement Geriatr Cogn Disord 2008;25:115-26.

9. American Psychiatric Association. Diagnostic and Statistical Manual of Mental Disorders (DSM-5). Amer Psychiatric Pub, Arlington, 2013.

10. Yu J, Li J, Huang X. The Beijing version of the Montreal Cognitive Assessment as a brief screening tool for mild cognitive impairment: a community-based study. BMC Psychiatry 2012;12:156.

11. Zhang ZX, Zahner GE, Roman GC et al. Sociodemographic variation of dementia subtypes in china: Methodology and results of a prevalence study in Beijing, Chengdu, Shanghai, and Xian. Neuroepidemiology 2006;27:177-87.

12. Katzman R, Zhang MY, Ouang Ya Q et al. A Chinese version of the Mini-Mental State Examination; impact of illiteracy in a Shanghai dementia survey. J Clin Epidemiol 1988;41:971-8. 
13. Barekatain M, Alavirad M, Tavakoli M et al. Cognitive rehabilitation in patients with nonamnestic mild cognitive impairment. J Res Med Sci 2016;21:101.

14. Buschert VC, Friese U, Teipel SJ et al. Effects of a newly developed cognitive intervention in amnestic mild cognitive impairment and mild Alzheimer's disease: a pilot study. J Alzheimers Dis 2011;25:679-94.

15. Kinsella GJ, Mullaly E, Rand E et al. Early intervention for mild cognitive impairment: a randomised controlled trial. J Neurol Neurosurg Psychiatry 2009;80:730-6.

16. Tsolaki M, Kounti F, Agogiatou C et al. Effectiveness of nonpharmacological approaches in patients with mild cognitive impairment. Neurodegener Dis 2011;8:138-45.

17. Loewenstein DA, Acevedo A, Czaja SJ et al. Cognitive rehabilitation of mildly impaired Alzheimer disease patients on cholinesterase inhibitors. Am J Geriatr Psychiatry 2004;12:395-402.

18. Albert MS, DeKosky ST, Dickson D et al. The diagnosis

Cite this article as: $\mathrm{Xu} \mathrm{S}$, Meng G, Chen P, Fang M, Jin A. Multidimensional intervention in individuals with mild cognitive impairment: a pilot nonrandomized study. Ann Palliat Med 2021;10(2):1539-1547. doi: 10.21037/apm-20-346 of mild cognitive impairment due to Alzheimer's disease: recommendations from the National Institute on AgingAlzheimer's Association workgroups on diagnostic guidelines for Alzheimer's disease. Alzheimers Dement 2011;7:270-9.

19. Gold DA. An examination of instrumental activities of daily living assessment in older adults and mild cognitive impairment. J Clin Exp Neuropsychol 2012;34:11-34.

20. Monastero R, Mangialasche F, Camarda C et al. A systematic review of neuropsychiatric symptoms in mild cognitive impairment. J Alzheimers Dis 2009;18:11-30.

21. Farias ST, Mungas D, Reed BR et al. Progression of mild cognitive impairment to dementia in clinic- vs communitybased cohorts. Arch Neurol 2009;66:1151-7.

22. Barnes DE, Santos-Modesitt W, Poelke G et al. The Mental Activity and eXercise (MAX) trial: a randomized controlled trial to enhance cognitive function in older adults. JAMA Intern Med 2013;173:797-804. 